\title{
Association between microbial characteristics and poor outcomes among patients with methicillin-resistant Staphylococcus aureus pneumonia: a retrospective cohort study
}

\author{
Jennifer S. McDanel ${ }^{1,2,3^{*}}$, Eli N. Perencevich ${ }^{1,2,3}$, Daniel J. Diekema ${ }^{2,4,5}$, Patricia L. Winokur ${ }^{2,3}$, J. Kristie Johnson ${ }^{6}$,
} Loreen A. Herwaldt ${ }^{1,2,5}$, Tara C. Smith ${ }^{1,8}$, Elizabeth A. Chrischilles ${ }^{1}$, Jeffrey D. Dawson ${ }^{7}$ and Marin L. Schweizer ${ }^{1,2,3}$

\begin{abstract}
Background: Methicillin-resistant S. aureus (MRSA) pneumonia is associated with poor clinical outcomes. This study examined the association between microbial characteristics and poor outcomes among patients with methicillin-resistant Staphylococcus aureus pneumonia.

Findings: This retrospective cohort study included 75 patients with MRSA pneumonia who were admitted to two large tertiary care medical centers during 2003-2010. Multivariable models were created using Cox proportional hazards regression and ordinal logistic regression to identify predictors of mortality or increased length of stay (LOS). None of the microbial characteristics (PFGE type, agr dysfunction, SCCmec type, and detection of PVL, ACME, and TSST-1) were significantly associated with 30-day mortality or post-infection hospital length of stay, after adjusting for gender, age, previous hospital admission within 12 months, previous MRSA infection or colonization, positive influenza test, Charlson Comorbidity Index score, and treatment (linezolid or vancomycin).
\end{abstract}

Conclusion: Large prospective studies are needed to examine the impact of microbial characteristics on the risk of death and other adverse outcomes among patients with MRSA pneumonia.

Keywords: MRSA pneumonia, Panton-Valentine leukocidin, Accessory Gene Regulator, mortality, length of stay

\section{Findings}

The literature is inconsistent regarding the association between microbial characteristics and poor outcomes among patients with methicillin-resistant Staphylococcus aureus (MRSA) pneumonia. Prior studies have linked the Panton-Valentine leukocidin (PVL) toxin and accessory gene regulator (agr) locus with necrosis of the lung $[1,2]$. Additionally, some antimicrobials,

\footnotetext{
* Correspondence: jennifer-mcdanel@uiowa.edu

Presented In Part: IDWeek, San Francisco, CA October 2-6, 2013. Abstract 41060.

'Department of Epidemiology, College of Public Health, University of lowa, lowa City, IA, USA

2Department of Internal Medicine, Carver College of Medicine, University of lowa, lowa City, IA, USA

Full list of author information is available at the end of the article
}

such as linezolid or clindamycin, used to treat MRSA pneumonia may inhibit specific microbial virulence mechanisms [3, 4]. This study evaluated a cohort of patients with MRSA pneumonia to assess the association between MRSA microbial characteristics and poor outcomes such as death or increased hospital length of stay (LOS).

This retrospective study included patients admitted to the University of Iowa Hospitals and Clinics (UIHC) or to the University of Maryland Medical Center (UMMC) during 2003-2010. Patients were initially identified by International Classification of Diseases, $9^{\text {th }}$ Revision, Clinical Modification (ICD-9-CM) codes for influenzalike illness [5] and were included if they had an ICD-9CM code for pneumonia (ICD-9-CM codes: 480-488, 
V12.61, 997.31), had a banked MRSA isolate from either a respiratory or a blood culture during their admissions, and began antibiotic therapy with vancomycin or linezolid within 2 days before until fourteen days after the collection of the first MRSA positive culture. This study was approved by the institutional review board of the University of Iowa.

Antimicrobial susceptibility testing was performed using broth dilution as described by the Clinical and Laboratory Standards Institute [6]. Isolates were tested for susceptibility to tetracycline, trimethoprim-sulfamethoxazole, tigecycline, levofloxacin, linezolid, daptomycin, and vancomycin. Heterogeneous vancomycin-intermediate $S$. aureus (hVISA) screen testing was performed according to previously published methods [7]. The methods for pulsed-field gel electrophoresis (PFGE) are previously described, and a similarity coefficient of $80 \%$ determined genetic relatedness $[8,9]$. The $a g r$ screening test was performed by measuring the expression of $\delta$-hemolysin using a $\beta$-lysin disk [10]. Polymerase chain reaction testing was used to identify the Panton-Valentine leukocidin (PVL) gene $[9,11]$, toxic shock syndrome toxin-1 (TSST-1) gene $[9,12]$, arginine catabolic mobile element (ACME) $[9,13]$, and staphylococcal cassette chromosome mec (SCCmec) [9] types I to V.

The primary outcomes analyzed were 30-day allcause in-hospital mortality and post-infection hospital LOS. Thirty-day mortality was defined as death occurring within 30 days after the collection of the

Table 1 Unadjusted associations for mortality or post-infection length of stay among patients with MRSA pneumonia

\begin{tabular}{|c|c|c|c|c|c|c|}
\hline \multirow[b]{2}{*}{ Characteristics } & \multicolumn{2}{|c|}{$\begin{array}{l}\text { Proportion of patients with } \\
\text { characteristic }\end{array}$} & \multicolumn{2}{|c|}{ 30-Day-In-Hospital mortality ${ }^{a}$} & \multicolumn{2}{|c|}{ Post-infection length of stay ${ }^{b}$} \\
\hline & $\begin{array}{l}\text { Patients who } \\
\text { died } N=18\end{array}$ & $\begin{array}{l}\text { Patients who } \\
\text { survived } N=57\end{array}$ & $\begin{array}{l}\text { Hazards ratio ( } 95 \% \\
\text { Confidence Interval) }\end{array}$ & $P$-value & $\begin{array}{l}\text { Odds ratio ( } 95 \% \\
\text { Confidence Interval) }\end{array}$ & $P$-value \\
\hline \multicolumn{7}{|l|}{ Patient characteristics } \\
\hline Gender: Female & $9(50 \%)$ & $20(35 \%)$ & $1.81(0.72-4.57)$ & 0.201 & $1.44(0.63-3.30)$ & 0.385 \\
\hline Age $\geq 55$ years & & & $4.69(1.36-16.22)$ & 0.015 & $2.15(0.95-4.87)$ & 0.068 \\
\hline $\begin{array}{l}\text { Hospital admission within } \\
\text { previous } 12 \text { months }\end{array}$ & $6(33 \%)$ & 31 (54 \%) & $0.52(0.19-1.37)$ & 0.185 & $0.40(0.17-0.91)$ & 0.028 \\
\hline $\begin{array}{l}\text { Previous MRSA infection or } \\
\text { colonization }\end{array}$ & $6(33 \%)$ & $11(19 \%)$ & $1.82(0.68-4.88)$ & 0.234 & $1.31(0.50-3.42)$ & 0.583 \\
\hline Positive influenza test & $2(11 \%)$ & $3(5 \%)$ & $1.69(0.39-7.38)$ & 0.488 & $2.13(0.41-11.01)$ & 0.368 \\
\hline $\begin{array}{l}\text { Charlson comorbidity index } \\
\text { score- median (IQR) }\end{array}$ & $2(1-3)$ & $2(1-4)$ & $0.99(0.83-1.20)$ & 0.948 & $0.99(0.84-1.16)$ & 0.896 \\
\hline Hospital-acquired infection & $6(33 \%)$ & $19(33 \%)$ & $0.85(0.32-2.29)$ & 0.752 & $1.50(0.64-3.53)$ & 0.354 \\
\hline \multicolumn{7}{|l|}{ Antimicrobial prescribed } \\
\hline Linezolid & $2(11 \%)$ & $12(21 \%)$ & $0.60(0.14-2.60)$ & 0.492 & $0.36(0.13-1.03)$ & 0.056 \\
\hline Vancomycin & 17 (94 \%) & $54(95 \%)$ & $0.61(0.08-4.68)$ & 0.223 & $3.10(0.51-19.02)$ & 0.221 \\
\hline Both linezolid and vancomycin & $1(6 \%)$ & $9(16 \%)$ & $0.37(0.05-2.76)$ & 0.331 & $0.43(0.13-1.41)$ & 0.162 \\
\hline \multicolumn{7}{|l|}{ Microbial characteristics } \\
\hline Panton-Valentine leukocidin & $5(28 \%)$ & $24(42 \%)$ & $0.82(0.36-1.88)$ & 0.645 & $0.82(0.36-1.88)$ & 0.645 \\
\hline \multicolumn{7}{|l|}{ sccmec } \\
\hline Type ॥ & $12(67 \%)$ & $27(47 \%)$ & $1.22(0.54-2.72)$ & 0.634 & $1.22(0.54-2.72)$ & 0.634 \\
\hline Type IV & $6(33 \%)$ & $29(51 \%)$ & $0.79(0.35-1.76)$ & 0.555 & $0.79(0.35-1.76)$ & 0.555 \\
\hline agr dysfunction & $4(22 \%)$ & $9(16 \%)$ & $1.35(0.44-4.10)$ & 0.598 & $1.59(0.54-4.62)$ & 0.399 \\
\hline $\begin{array}{l}\text { Arginine catabolic mobile } \\
\text { element }\end{array}$ & $3(17 \%)$ & $22(39 \%)$ & $0.35(0.10-1.20)$ & 0.094 & $0.70(0.30-1.64)$ & 0.413 \\
\hline Toxic shock syndrome toxin-1 & $1(6 \%)$ & $2(4 \%)$ & $1.22(0.16-9.19)$ & 0.846 & $2.35(0.29-19.27)$ & 0.423 \\
\hline \multicolumn{7}{|l|}{ PFGE type } \\
\hline USA100 & $11(61 \%)$ & $25(44 \%)$ & $1.87(0.72-4.82)$ & 0.197 & $1.20(0.54-2.69)$ & 0.652 \\
\hline USA300 & $4(22 \%)$ & $25(44 \%)$ & $0.40(0.13-1.21)$ & 0.104 & $0.66(0.29-1.51)$ & 0.323 \\
\hline Other & $3(17 \%)$ & $7(12 \%)$ & $1.40(0.41-4.85)$ & 0.594 & $1.57(0.48-5.16)$ & 0.458 \\
\hline
\end{tabular}

$I Q R$ interquartile range, $S C C m e c$ staphylococcal cassette chromosome mec, agr accessory gene regulator, PFGE pulsed-field gel electrophoresis, $h V I S A$ heterogeneous vancomycin-intermediate $S$. aureus

${ }^{a}$ Defined as death occurring within the first 30 days after the day when the first MRSA positive respiratory or blood culture was collected

${ }^{b}$ Defined as the day the first MRSA positive respiratory or blood culture was collected until the day the patient was either discharged from the hospital or died 
Table 2 Adjusted associations for mortality or post-infection length of stay among patients with MRSA pneumonia ${ }^{a}$

\begin{tabular}{|c|c|c|c|c|}
\hline \multirow[b]{2}{*}{ Microbial characteristics } & \multicolumn{2}{|l|}{ 30-Day-In-Hospital mortality ${ }^{b}$} & \multicolumn{2}{|l|}{ Post-infection length of stay ${ }^{c}$} \\
\hline & Hazards ratio (95 \% Confidence Interval) & $P$-value & Odds ratio (95 \% Confidence Interval) & $P$-value \\
\hline Panton-Valentine leukocidin & $0.72(0.23-2.25)$ & 0.566 & $0.67(0.27-1.69)$ & 0.398 \\
\hline \multicolumn{5}{|l|}{ sccmec } \\
\hline Type ॥ & $1.66(0.53-5.16)$ & 0.383 & $1.77(0.69-4.52)$ & 0.235 \\
\hline Type IV & $0.62(0.20-1.93)$ & 0.414 & $0.51(0.20-1.30)$ & 0.158 \\
\hline agr dysfunction & $1.84(0.54-6.20)$ & 0.323 & $1.30(0.42-4.05)$ & 0.648 \\
\hline Arginine catabolic mobile element & $0.37(0.10-1.47)$ & 0.158 & $0.57(0.22-1.48)$ & 0.250 \\
\hline Toxic shock syndrome toxin-1 & $1.24(0.14-11.10)$ & 0.847 & $2.86(0.26-31.18)$ & 0.389 \\
\hline \multicolumn{5}{|l|}{ PFGE type } \\
\hline USA100 & $1.29(0.42-3.95)$ & 0.656 & $1.70(0.67-4.33)$ & 0.268 \\
\hline USA300 & $0.54(0.15-1.92)$ & 0.344 & $0.60(0.24-1.50)$ & 0.269 \\
\hline
\end{tabular}

IQR interquartile range, SCCmec staphylococcal cassette chromosome mec, agr accessory gene regulator, PFGE pulsed-field gel electrophoresis

${ }^{a}$ Adjusted for gender, age, previous hospital admission within 12 months, previous MRSA infection or colonization, positive influenza test, Charlson Comorbidity Index score, and treatment (linezolid or vancomycin)

${ }^{b}$ Defined as death occurring within the first 30 days after the day when the first MRSA positive respiratory or blood culture was collected

${ }^{c}$ Defined as the day the first MRSA positive respiratory or blood culture was collected until the day the patient was either discharged from the hospital or died

Table 3 Patient characteristics, microbial characteristics, or treatments associated with strain type $(N=65)$

\begin{tabular}{|c|c|c|c|}
\hline Characteristics & USA100 N=36 & USA300 N = 29 & $P$-value \\
\hline \multicolumn{4}{|l|}{ Patient characteristics } \\
\hline Gender: Female & $16(44 \%)$ & $9(31 \%)$ & 0.269 \\
\hline Age $\geq 55$ years & $24(67 \%)$ & $11(38 \%)$ & 0.021 \\
\hline Hospital admission within previous 12 months & $21(58 \%)$ & $11(38 \%)$ & 0.102 \\
\hline Hospital-acquired infection & $10(28 \%)$ & $12(41 \%)$ & 0.249 \\
\hline Previous MRSA infection or colonization & $8(22 \%)$ & $5(17 \%)$ & 0.618 \\
\hline Positive influenza test & $1(3 \%)$ & $2(7 \%)$ & 0.582 \\
\hline Post-infection length of stay: median (IQR), in days ${ }^{a}$ & $6(2-18)$ & $12(4-24)$ & 0.232 \\
\hline Charlson Comorbidity Index score: median (IQR) & $2(1-3)$ & $2(0-4)$ & 0.778 \\
\hline 30-day mortality ${ }^{\mathrm{b}}$ & $11(31 \%)$ & $4(14 \%)$ & 0.111 \\
\hline \multicolumn{4}{|l|}{ Antimicrobial prescribed } \\
\hline Linezolid & $11(31 \%)$ & $2(7 \%)$ & 0.018 \\
\hline Vancomycin & $33(92 \%)$ & $29(100 \%)$ & 0.247 \\
\hline \multicolumn{4}{|l|}{ Microbial characteristics } \\
\hline agr dysfunction & $8(22 \%)$ & $4(14 \%)$ & 0.384 \\
\hline Toxic shock syndrome toxin-1 & $1(3 \%)$ & $0(0 \%)$ & 1.000 \\
\hline hVISA & $1(3 \%)$ & $0(0 \%)$ & 1.000 \\
\hline Arginine catabolic mobile element & $1(3 \%)$ & $23(79 \%)$ & $<0.001$ \\
\hline Panton-Valentine leukocidin & $0(0 \%)$ & $26(90 \%)$ & $<0.001$ \\
\hline \multicolumn{4}{|l|}{ sccmec } \\
\hline Type ॥ & $35(97 \%)$ & $4(14 \%)$ & $<0.001$ \\
\hline Type IV & $0(0 \%)$ & $25(86 \%)$ & $<0.001$ \\
\hline
\end{tabular}

$I Q R$ interquartile range, $S C C m e c$ staphylococcal cassette chromosome mec, agr accessory gene regulator, PFGE pulsed-field gel electrophoresis, $h V I S A$ heterogeneous vancomycin-intermediate $S$. aureus

${ }^{a}$ Defined as the day the first respiratory or blood culture that grew MRSA was collected until the day the patient was either discharged from the hospital or died

${ }^{b}$ Defined as death occurring within the first 30 days after the day when the first respiratory or blood culture that grew MRSA was obtained 
first MRSA positive respiratory or blood culture. Hospital LOS was measured beginning on the day the first MRSA positive respiratory or blood culture was collected until either hospital discharge or death. Comorbidities were measured using the Charlson Comorbidity Index [14]. Age was dichotomized on the median. Information was collected on hospital admission within the previous 12 months, previous MRSA infection or colonization, and having a positive influenza test during hospital admission. Hospital-acquired infections were defined as the first MRSA positive respiratory or blood culture collected more than 2 days after hospital admission.

Bivariable analyses were conducted using either Student's t-test or the Wilcoxon Rank Sum test for continuous variables and either the chi-square test or Fisher's exact test for categorical variables. Cox proportional hazard regression and ordinal logistic regression were used to perform the multivariable analyses assessing the association between microbial factors and 30-day mortality or LOS. Patients who did not die were categorized based on their length of stay: 0-3 days, 4-10 days, 11-20 days, and $\geq 21$ days. Deceased patients were placed in a separate category since they had the worst outcome and varying lengths of stay before death. Variables with $P<0.25$ in the bivariable analysis were entered into the model using a manual stepwise method, and remained in the multivariable model if they were statistically significant $(P<0.05)$. Data were analyzed using SAS software (SAS Institute, Cary, NC) version 9.3.

The cohort was comprised of 75 patients with MRSA pneumonia. The majority of patients were male (61\%) and the median age was 54 . Twenty-four percent (18/75) of the patients died and the median post-infection LOS in the hospital was 9 days (interquartile range: 3-20). Most isolates were from respiratory cultures, including bronchial washes (9\%), tracheal aspirates (7\%), and sputum cultures (83\%).

Patients who survived were more likely than patients who died to be infected with MRSA isolates that were PVL-positive (42\% vs. $28 \%$; $P=0.277$ ) or ACME-positive (39 \% vs. $17 \% ; P=0.085)$. Patients who died were more likely than those who survived to be infected with MRSA isolates with a dysfunctional $\operatorname{agr}(22 \%$ vs. $16 \% ; P=0.530)$. However, none of these results reached statistical significance (Table 1). Most MRSA isolates were susceptible to all antimicrobials tested except levofloxacin (9\% susceptible). All isolates were susceptible to vancomycin [minimum inhibitory concentration (MIC) range: $0.5-1 \mu \mathrm{g} / \mathrm{mL}$ ] and linezolid (MIC range: $0.5-2$ $\mu \mathrm{g} / \mathrm{mL}$ ) [Additional file: Susceptibility to antimicrobial agents of MRSA isolates from patients with MRSA pneumonia $(N=75)$ (see Additional file 1)]. None of the microbial characteristics were statistically significantly associated with increased post-infection LOS or mortality in the multivariable analyses statistically adjusting for gender, age, previous hospital admission within 12 months, previous MRSA infection or colonization, positive influenza test, Charlson Comorbidity Index score, and treatment (linezolid or vancomycin) (Table 2).

Sixty-five patients $(87 \%)$ were infected with either a USA300 strain or a USA100 strain. Patients infected with a USA300 strain were less likely to receive linezolid (7 \% vs. $31 \% ; P=0.018$ ) and were more likely to be younger ( $<55$ years) [38 \% vs. $67 \% ; P=0.021$ ] compared with patients infected with a USA100 strain (Table 3). Additionally, $14 \%$ of the patients infected with a USA300 strain died compared with $31 \%$ of the patients infected with a USA100 strain $(P=0.111)$.

Numerous investigators have tried to determine whether specific microbial characteristics of MRSA infecting isolates are associated with poor outcomes $[1,2$, $15,16]$. However, the results of those studies vary substantially. Even though this study was larger than most studies to examine microbial characteristics associated with MRSA pneumonia, this study did not identify any statistically significant associations between the microbial characteristics and outcomes. Even though the risk estimates suggested an association between a few of the microbial characteristics and death or increased postinfection LOS, the confidence intervals were wide, potentially due to the limited sample size and small number of patients who died. However, among patients infected with USA100 strains or USA300 strains, there was a trend toward increased mortality among those infected with USA100 strains.

Our study had limitations. First, patients might have been colonized in the throat or upper respiratory tract rather than having MRSA respiratory infections. To identify MRSA infected patients, this study only included patients who received vancomycin or linezolid (agents active against MRSA). Second, influenza-like illness ICD-9-CM codes were used to identify the study population because the patients initially were included in a study of influenzalike illness and MRSA pneumonia, thus this cohort may be subject to selection bias. However, this is not a large concern since cough was the most common influenza-like illness code identified. Finally, since this is a retrospective study, patients who had pneumonia but did not have a culture collected during their admission would not be included in the study. Large prospective studies are needed to assess whether microbial factors in combination with treatment factors or patient factors increase the risk of death and other adverse outcomes. 


\section{Additional file}

\section{Additional file 1. Susceptibility to antimicrobial agents of MRSA} isolates from patients with MRSA pneumonia $(N=75)$. The

susceptibility results for the 75 isolates that were tested to tetracycline, trimethoprim-sulfamethoxazole, tigecycline, levofloxacin, linezolid, daptomycin, and vancomycin. (DOCX $14 \mathrm{~kb}$ )

\section{Competing interests}

ENP, PLW, JKJ, LAH, TCS, EAC, and JDD declare that they have no competing interests relevant to this article. JSM has received speaker honorarium from Biomerieux. DJD has received research funding from Cerexa, a subsidiary of Forest Laboratories. MLS received the Young Investigator Award from Pfizer

\section{Authors' contributions}

JSM performed the laboratory testing, analyzed the data, and wrote the manuscript. ENP helped design the study, collaborated between centers, interpreted the results from the regression analysis, and revised the manuscript. PLW helped design the study, identified the patient populations, and revised the manuscript. JKJ coordinated the collection and shipment of the laboratory specimens, extracted the patient data from the UMMC, and revised the manuscript. LAH, TCS, and EAC aided in interpreting the study results and revised the manuscript. JDD assisted in the data analysis, interpreted the results from the regression analysis, and revised the manuscript. DJD helped design the study, aided in collecting specimens from $\mathrm{UIHC}$, provided laboratory support for testing the isolates, interpreted results, and revised the manuscript. MLS helped design the study, collected specimens and patient data from both centers, collaborated between centers, identified the patient population, interpreted results, and revised the manuscript. All authors read and approved the final manuscript.

\section{Acknowledgments}

Financial support: The study was funded in part by a Young Investigator Award from Pfizer (\#WS79560E). MLS is funded by a VA HSR\&D Career Development Award (CDA 11-211). ENP was funded through a VA HSR\&D grant (IIR 09-099). JSM was supported by a grant from Cubist Pharmaceuticals.

Role of Funding Source: Pfizer staff members did not participate in the design or conduct of the study or in the manuscript preparation. JSM takes full responsibility for the integrity of this study.

\section{Author details}

'Department of Epidemiology, College of Public Health, University of lowa, lowa City, IA, USA. '2Department of Internal Medicine, Carver College of Medicine, University of lowa, lowa City, IA, USA. ${ }^{3}$ lowa City Veterans Affairs Health Care System, lowa City, IA, USA. ${ }^{4}$ Department of Pathology, Carver College of Medicine, University of lowa, lowa City, IA, USA. ${ }^{5}$ Clinical Quality, Safety, and Performance Improvement, University of lowa Hospitals and Clinics, lowa City, IA, USA. ${ }^{6}$ Department of Pathology, University of Maryland School of Medicine, Baltimore, MD, USA. ${ }^{7}$ Department of Biostatistics, College of Public Health, University of lowa, lowa City, IA, USA. ${ }^{8}$ Present address: Department of Biostatistics, Environmental Health Sciences, and Epidemiology, Kent State University, Kent, OH, USA.

Received: 27 July 2015 Accepted: 12 November 2015

Published online: 14 December 2015

\section{References}

1. Diep BA, Chan L, Tattevin P, Kajikawa O, Martin TR, Basuino L, et al. Polymorphonuclear leukocytes mediate Staphylococcus aureus PantonValentine leukocidin-induced lung inflammation and injury. Proc Natl Acad Sci U S A. 2010;107:5587-92.

2. Montgomery CP, Boyle-Vavra S, Daum RS. Importance of the global regulators Agr and SaeRS in the pathogenesis of CA-MRSA USA300 infection. PLoS One. 2010;5:e15177.

3. Bernardo K, Pakulat N, Fleer S, Schnaith A, Utermohlen O, Krut O, et al. Subinhibitory concentrations of linezolid reduce Staphylococcus aureus virulence factor expression. Antimicrob Agents Chemother. 2004;48:546-55.

4. Micek ST, Dunne M, Kollef MH. Pleuropulmonary complications of Panton-Valentine leukocidin-positive community-acquired methicillin- resistant Staphylococcus aureus: importance of treatment with antimicrobials inhibiting exotoxin production. Chest. 2005;128:2732-8.

5. Marsden-Haug N, Foster VB, Gould PL, Elbert E, Wang H, Pavlin JA. Code-based syndromic surveillance for influenza like illness by International Classification of Diseases, Ninth Revision. Emerg Infect Dis. 2007;13:207-16.

6. Clinical and Laboratory Standards Institute. Methods for dilution antimicrobial susceptibility tests for bacteria that grow aerobically. Approved standard M07-A8. Wayne: Clinical and Laboratory Standards Institute; 2009.

7. Satola SW, Farley MM, Anderson KF, Patel JB. Comparison of detection methods for heteroresistant vancomycin-intermediate Staphylococcus aureus, with the population analysis profile method as the reference method. J Clin Microbiol. 2011;49:177-83.

8. Pfaller MA, Hollis RJ, Sader HS. Chromosomal restriction fragment analysis by pulsed field gel electrophoresis. In: Isenberg HD, editor. Clinical microbiology procedures handbook. Supplement 1. Washingtonw: American Society for Microbiology; 1994. p. 10.5c1-10.5c12.

9. Richter SS, Heilmann KP, Dohrn CL, Riahi F, Costello AJ, Kroeger JS, et al. Activity of ceftaroline and epidemiologic trends in Staphylococcus aureus isolates collected from 43 medical centers in the United States in 2009. Antimicrob Agents Chemother. 2011;55:4154-60.

10. Traber KE, Lee E, Benson S, Corrigan R, Cantera M, Shopsin B, et al. agr function in clinical Staphylococcus aureus isolates. Microbiology. 2008;154: 2265-74.

11. Lina G, Piemont Y, Godail-Gamot F, Bes M, Peter MO, Gauduchon V, et al. Involvement of Panton-Valentine leukocidin-producing Staphylococcus aureus in primary skin infections and pneumonia. Clin Infect Dis. 1999;29: $1128-32$.

12. Mehrotra M, Wang G, Johnson WM. Multiplex PCR for detection of genes for Staphylococcus aureus enterotoxins, exfoliative toxins, toxic shock syndrome toxin 1, and methicillin resistance. J Clin Microbiol. 2000;38:1032-5.

13. Diep BA, Gill SR, Chang RF, Phan TH, Chen JH, Davidson MG, et al. Complete genome sequence of USA300, an epidemic clone of community-acquired methicillin-resistant Staphylococcus aureus. Lancet. 2006;367:731-9.

14. Deyo RA, Cherkin DC, Ciol MA. Adapting a clinical comorbidity index for use with ICD-9-CM administrative databases. J Clin Epidemiol. 1992;45:613-9.

15. Sharma-Kuinkel BK, Ahn SH, Rude TH, Zhang Y, Tong SY, Ruffin F, et al. Presence of genes encoding Panton-Valentine Leukocidin is not the primary determinant of outcome in patients with hospital-acquired pneumonia due to Staphylococcus aureus. J Clin Microbiol. 2012;50:848-56.

16. Schweizer ML, Furuno JP, Sakoulas G, Johnson JK, Harris AD, Shardell MD, et al. Increased mortality with accessory gene regulator (agr) dysfunction in Staphylococcus aureus among bacteremic patients. Antimicrob Agents Chemother. 2011;55:1082-7.

\section{Submit your next manuscript to BioMed Central and take full advantage of:}

- Convenient online submission

- Thorough peer review

- No space constraints or color figure charges

- Immediate publication on acceptance

- Inclusion in PubMed, CAS, Scopus and Google Scholar

- Research which is freely available for redistribution 